\title{
Prediction of Hazard Identification and Characterization of Several Compounds used as Food Additives Applying In Silico Methods
}

\author{
Iltizam Nasrullah $^{1}$, Rahmana Emran Kartasasmita ${ }^{2 *}$, Sophi Damayanti ${ }^{2}$ \\ ${ }^{I}$ National Agency of Drug and Food Control of the Republic of Indonesia, Jl. Percetakan Negara No 23, Jakarta 10560, Indonesia \\ ${ }^{2}$ School of Pharmacy Bandung Institute of Technology, Labtek VII, Ganesha 10 Bandung 40132, Indonesia
}

Received: 30 September 2014 / Accepted: 30 November 2014

\begin{abstract}
:
In frame of risk analysis, hazard identification and characterization are parts of risk assessment that should be performed to assure the safety aspect of a substance that will be used as food additive. According to WHO guidance, the two steps can be performed experimentally, based on epidemiological data or based on chemical structure calculation. In line with this guidance, the aim of this research was to obtain data describe the hazardous properties of several compounds used as food additive applying predictive in silico methods. Hazardous properties were limited to mutagenicity and carcinogenicity effects. The software applied including Toxtree, T.E.S.T., VEGA Benigni/Bossa, VEGA CAESAR and VEGA SARpy. A positive result can be accepted if all the prediction models give the same results and alerts on the compound for carcinogenic and mutagenic potential. If only one or more, but not all those prediction models, showing alert on a compound or give positive result, than it is assumed that the results are still not enough to predict carcinogenic or mutagenic effects of that compound unless experimental data supporting those effects are available. Fifty four compounds of flavour functional class were included in our study. Following our criteria, 20 compounds of them show negative results on mutagenicity prediction; however the rests 34 compounds give different prediction results and hence need experimental data for confirmation. In the case of carcinogenicity predictions, 14 compounds of them were predicted to show no carcinogenic potential, 10 compounds of them were predicted to show carcinogenic potential, while the rests 30 compounds show different prediction results and hence need experimental data for confirmation. Based on overall results, it was concluded that this in silico approaches can be used to perform preliminary evaluation of hazardous properties, especially those of mutagenicity and carcinogenicity effects.
\end{abstract}

Key words: Food additives, in silico methods, hazardous properties, risk analysis

\section{Introduction}

Hazard identification and characterization are parts of risk assessment that should be performed to assure the safety aspect of a substance that will be used as food additive. When a risk assessment is performed, there are often insufficient data available to complete the assignment. Scientific information can be used to support many food safety risk assessments and it is available from a variety of sources, both national and international. According to WHO guidance, hazard identification and characterization can be performed experimentally, based on epidemiological data, or based on chemical structure calculation / prediction [1]. Mutagenicity and carcinogenicity tests are important part of the hazard assessment for food additives.

Flavour is one of the food additives functional classes that can give consumer perception about the food they consume. Food having attractive flavour will be the one that a consumer chooses. In Indonesia, 1828 compounds are permitted as flavouring substances [2]. Most of them are included in the lists of JECFA, EC and FEMA. However some compounds are also included in the list of IARC as $2 \mathrm{~A}$ or $2 \mathrm{~B}$ groups. Some listed flavours belong to compound group with high chemical reactivities but despite these reactivities, they are usually lack of toxicity data. These very limited toxicity data makes difficult to an agreement on safety level concern and consequently the development of management options was challenging [3].

Currently, the interest to apply in silico methods for toxicity screening of a compound has increased. These methods were considered useful only for providing a rough and initial estimation, but now it has attracted interest as they can guide the sciencetist to investigate toxic potential [4]. The application of in silico approaches for predicting preclinical toxicological endpoints, clinical adverse effects, and metabolism of pharmaceutical substances has become of high interest to the scientific community and the public [5]. In silico / computational approaches can be used to study toxicity of a compound based on its molecular structure and properties predicted from similar compounds whose biological activities are known [6]. The balances between experimental methods and in silico / computational approaches are expected to reduce the number of safety issues [7]. Computational toxicological prediction is needed to reduce cost and time, animal testing and to develop legislation related with the use of

\footnotetext{
*Corresponding author: Rahmana Emran Kartasasmita,

E-mail: emran_kartasasmita@yahoo.de
} 
new substances [8]. Several in silico tools are available for toxicity prediction of a substance, such as Toxtree, T.E.S.T., VEGA Benigni/Bossa, VEGA CAESAR etc. They have been tuned to predict global toxicity endpoints, such as mutagenicity and carcinogenicity.

The aim of this study was to obtain data described the hazardous properties of several compounds used as food additive, especially of flavour functional class, applying predictive in silico methods. Hazardous properties were limited to mutagenicity and carcinogenicity effects.

\section{Experimental}

\section{Computational Software}

Computational analysis was performed applying the following softwares: TOXTREE (version 2.6.0), T.E.S.T. (version 4.1), VEGA Benigni/Bossa (version 1.0.0DEV), VEGA CAESAR (version 2.1.12 for mutagenicity and 2.1.8 for carcinogenicity) and VEGA SARpy (version 1.0.6-DEV). All these softwares can be used to predict mutagenicity and/or carcinogenicity end point. Toxtree is rule based software, while T.E.S.T. and CAESAR are statistical based softwares and SARpy is rule/statistical based software [9, 10]. VEGA Benigni/Bossa uses similar prediction model as Toxtree.

\section{Tested compound}

Thirty seven aromatic aldehydes and 17 furan derivatives are included in the study. The 37 aromatic aldehydes are p-tolylacetaldehyde, p-isopropyl phenylacetaldehyde, cinnamaldehyde, 3-(pisopropylphenyl) propionaldehyde, alpha-amyl cinnamaldehydedimethyl-acetal, p-methyl cinnamaldehyde, alpha-methyl cinnamaldehyde, alphabuthylcinnamaldehyde, alpha-amyl cinnamaldehyde, alpha-hexyl cinnamaldehyde, p-methoxycinnamaldehyde, o-methoxy-cinnamaldehyde, p-methoxy-alphamethylcinnamaldehyde, 2-methyl-4-phenylbutyraldehyde, 3methyl-2-phenylbutyraldehyde, 2-methyl-3-(p-isopropylphenyl) propionaldehyde, 2-methyl-3-tolyl propionaldehyde, 2-phenyl propionaldehyde, 2-phenyl propionaldehyde dimethyl acetal, 2-(p-tolyl) propionaldehyde, benzaldehyde, benzaldehyde dime-thyl acetal, benzaldehydeglycerylacetal, benzaldehyde propylene glycol acetal, 4-ethylbenzaldehyde, tolualdehyde, tolualdehydeglycerylacetal, cuminaldehyde, 2,4-dimethyl-benzaldehyde, veratraldehyde, pmethoxybenzaldehyde, p-ethoxybenzaldehyde, salicylaldehyde, 2-hydroxy-4-methyl-benzaldehyde, 4-hydroxy benzaldehyde, o-methoxybenzaldehyde and 4-hydroxy3,5-dimethoxy-benzaldehyde.

Seventeen furan derivates are 2benzofurancarboxaldehyde, 2-furfurylidenebutyraldehyde, 2-methylfuran, 2,5-dimethylfuran, 2-ethylfuran, 2butylfuran, 2-pentylfuran, 2-heptylfuran, 2-decylfuran, 2-butyrylfuran, 2-pentanoylfuran, 3-methyl-2-(3methylbut-2-enyl)-furan, 2,3-dimethylbenzofuran, 2,4- difurfurylfuran, 2-acetyl-5-methylfuran, 2-acetyl-3,5dimethylfuran and 2-acetyl-2,5-dimethylfuran.

Nine compounds with known mutagenicity and carcinogenicity properties from experimental results are used as positive control. They are 2-naphthyl-amine, 4-(Nnitrosomethyl-amino)-1-(3-pyridyl)-1-butano-ne, 4amino biphenyl, benzidine, benzo[a]pyrene, bis (chloromethyl) ether, chloromethyl methyl ether, ethylene oxide and N-nitrosonor-nicotine [11].

\section{Methodology}

Prior application of computational softwares, all compounds were drawn and their geometry structures were optimized using Hyperchem (version 8.0.10), except for Toxtree and VEGA. These softwares need no geometry optimization but the structure of a compound must be first converted into SMILE notation prior calculation. The toxicity predictions were then carried out by means of existing rules available in those software. The following incusion and exclusion criteria are applied: If all softwares give negative results on carcinogenicity and mutagenicity predictions, then a compound is classified as show no carcinogenicity and / or mutagenicity potential. On the contrary if all softwares give positive results on carcinogenicity and mutagenicity predictions, then a compound is classified as show carcinogenicity and/or mutagenicity potential. If these softwares give different / inconsistent results, then no classification of carcinogenicity and / or mutagenicity potential can be made. The last two cases need experimental data for confirmation.

\section{Results and Discussion}

\section{Mutagenicity and Carcinogenicity}

All positive control compounds show mutagenic potential / structural alert for their mutagenicity prediction when predicted using all softwares, except bis(chloromethyl)-ether and chloromethylmethylether for SARPy model as well as N-nitrosonornicotine for VEGA Benigni/Bossa test model. The results of positive control compounds are displayed in Table 1 . In the case of tested compounds, 20 compounds of them show no mutagenicity prediction / no structural alert for mutagenicity when predicted using all softwares. However 34 compounds of them show different results when predicted using the same softwares and hence need experimental data for confirmation. Mutagenicity prediction results of tested compounds are summarized in Table 2 .

Carcinogenicity predictions of all compounds were performed applying Toxtree, VEGA Benigni/Bossa, and CAESAR. All positive control compounds show carcinogenic/have structural alert for their carcinogenicity when predicted using all the software, except N-nitrosonornicotine when tested with VEGA Benigni/Bossa test model. The results of positive control compounds are displayed in Table 3. 
In the case of tested compounds, 14 compounds of them show no carcinogenicity prediction / no structural alert for carcinogenicity, 10 compounds of them show carcinogenicity prediction / structural alert for carcinogenicity when predicted using all softwares, while the rests (30 compounds) show different results when predicted using the same softwares and hence need experimental data for confirmation. Furthermore confirmation from experimental data is also necessary for the 10 positive compounds. Mutagenicity prediction results of tested compounds are summarized in Table 4.

Test models of mutagenicity and carcinogenicity available in the softwares applied were actually already compared and tested by other researchers. It is already count for its specificity, selectivity and accuracy. It is stated that the best prediction result was obtained by software using statistical model $[9,10,12]$.

In our study, three of five softwares are statistical based software. However, due to differences in results among these softwares, it was still difficult to make a final decision.

Following that criteria, 20 compounds showed negative results on mutagenicity prediction; however 34 compounds need experimental data for confirmation. 10 compounds were predicted to have carcinogenic potential, while 14 compounds showed negative results on carcinogenicity prediction; however 40 compounds need experimental data for confirmation.

The application and acceptability of this in silico assessment can be used in other food additives and to perform preliminary evaluation of hazardous properties, especially those of mutagenicity and carcinogenicity effects.

The main problem of in silico prediction application in the risk assessment of food additives, especially for hazard identification and characterization of a food additive is difficulties in making decision. For this reasons, application and acceptability of in silico result in this field is still limited only for performing preliminary evaluation of hazardous properties, especially those of mutagenicity and carcinogenicity effects. Despite these limitations and difficulties, in silico methods can be very useful when the experimental data are not available or when the experimental tests are very difficult or practically impossible to be carried out.

\section{Conclusions}

Following our criteria, from total 54 tested compounds, 20 compounds of them show negative results on mutagenicity predictions; the rests 34 compounds give different prediction results and hence need experimental data for confirmation. In the case of carcinogenicity predictions, 14 compounds of them were predicted to show no carcinogenic potential, 10 compounds of them were predicted to show carcinogenic potential, while the rests 30 compounds show different prediction results. Consequently, 40 tested compounds still need experimental data on their carcinogenicities for confirmation. Based on overall results, it was concluded that despite the need of experimental data, in silico approaches can be used to perform preliminary evaluation of hazardous properties, especially those of mutagenicity and carcinogenicity effects.

\section{References}

[1] WHO-FAO, Food Safety Risk Analysis. A guide for national food safety authorities, FAO Food and Nutrition Paper, 87, 2006, 37-60.

[2] SNI, Standar Nasional Indonesia Bahan Tambahan PanganPersyaratan Perisa dan Penggunaan dalam Produk Pangan, 2006 (SNI) 01-7152-2006.

[3] B. Schilter, R. Benigni, A. Boobis, A. Chiodini, A. Cockburn, M.T.D. Cronin, E.L. Piparo, S. Modi, A. Thiel, A. Worth, Establishing the level of safety concern for chemicals in food without the need for toxicity testing, Regulatory Toxicology and Pharmacology, 68, 2014, 275-296.

[4] A. Ronclagioni, A.A. Toropov, A.P. Toropova, E. Benfenati, Insilico methods to predict drug toxicity, Current Opinion in Pharmacology, 13, 2013, 802-806.

[5] L.G. Valerio Jr., In silico toxicology for the pharmaceutical sciences, Toxicology and Applied Pharmacology, 241, 2009, 356-370

[6] E.L. Piparo, A. Worth, M. Manibusan, C. Yang, B. Schilter, P. Mazzatorta, M.N. Jacobs, H. Steinkellner, L. Mohimont, Use of computational tools in the field of food safety, Regulatory Toxicology and Pharmacology, 60, 2011, 354362.

[7] C. Merlot, Computational Toxicology-A Tool for Early Safety Evaluation, Reviews, Drug Discovery Today, 2010, Volume 15, Numbers 1-2.

[8] A.A. Toropov, A.P. Toropova, I. Raska Jr, D. Leszczynska, J. Leszczynski, Comprehension of drug toxicity: Software and database, Computers in Biology and Medicine, 45, 2014, 2025

[9] N.G. Bahtyari, G. Raitano, E. Benfenati, T. Martin, D. Young, Comparison of in silico models for prediction of mutagenicity. Journal of Environmental Science and Health, Part C, 31, 2013, 45-66.

[10] C.Milan, N.G. Bakhtyari, A. Roncaglioni, A. Cassano, G. Raitano, E. Benfenati, Comparison of the results of QSAR models for Mutagenicity, 15 $5^{\text {th }}$ International Workshop on Quantitative Structure-Activity in Environmental and Health Sciences, Tallinn-Estonia, June $18^{\text {th }}-22^{\text {nd }} 2012$.

[11] IARC(International Agency for Research on Cancer), Agents Classified by the IARC Monographs, 2014, Volumes 1-109.

[12] A. Ronclagioni, C. Milan, O. Schifanella, E. Benfenati, Comparison and Use of QSAR software to Estimate Carcinogenicity, $1^{\text {th }}$ International Workshop on QSARS in Environmental and Health Sciences, Montreal-Canada, May $24^{\text {th }}-28^{\text {th }} 2010$. 
Table 1. Mutagenicity Prediction for Control Chemical

\begin{tabular}{|c|c|c|c|c|c|c|}
\hline No. & Compound & Toxtree & T.E.S.T. & $\begin{array}{l}\text { VEGA } \\
\text { Benigni/Bossa }\end{array}$ & CAESAR & SARpy \\
\hline 1 & Benzo[a]pyrene & $\begin{array}{l}\text { Structural alert for S. typhimurium } \\
\text { mutagenicity }\end{array}$ & $\begin{array}{l}\text { Mutagenicity } \\
\text { Positive }\end{array}$ & Mutagen & Mutagen & Mutagen \\
\hline 2 & Benzidine & $\begin{array}{l}\text { Structural alert for } S \text {. typhimurium } \\
\text { mutagenicity; Potensial } S \text {. } \\
\text { typhimurium TA } 100 \text { mutagen }\end{array}$ & $\begin{array}{l}\text { Mutagenicity } \\
\text { Positive }\end{array}$ & Mutagen & Mutagen & Mutagen \\
\hline 3 & $\begin{array}{l}\text { Bis (chloromethyl) } \\
\text { ether }\end{array}$ & $\begin{array}{l}\text { Structural alert for } S . \text { typhimurium } \\
\text { mutagenicity }\end{array}$ & $\begin{array}{l}\text { Mutagenicity } \\
\text { Positive }\end{array}$ & Mutagen & Mutagen & Non Mutagen \\
\hline 4 & $\begin{array}{l}\text { Chloromethyl } \\
\text { methyl ether }\end{array}$ & $\begin{array}{l}\text { Structural alert for S. typhimurium } \\
\text { mutagenicity }\end{array}$ & $\begin{array}{l}\text { Mutagenicity } \\
\text { Positive }\end{array}$ & Mutagen & Mutagen & Non Mutagen \\
\hline 5 & Ethylene oxide & $\begin{array}{l}\text { Structural alert for } S \text {. typhimurium } \\
\text { mutagenicity }\end{array}$ & $\begin{array}{l}\text { Mutagenicity } \\
\text { Positive }\end{array}$ & Mutagen & Mutagen & Mutagen \\
\hline 6 & $\begin{array}{l}\text { N'- } \\
\text { Nitrosonornicotine }\end{array}$ & $\begin{array}{l}\text { Structural alert for } S \text {. typhimurium } \\
\text { mutagenicity }\end{array}$ & $\begin{array}{l}\text { Mutagenicity } \\
\text { Positive }\end{array}$ & Non Mutagen & Mutagen & Mutagen \\
\hline 7 & 2-Naphthylamine & $\begin{array}{l}\text { Structural alert for } S . \text { typhimurium } \\
\text { mutagenicity; Potensial } S \text {. } \\
\text { typhimurium TA } 100 \text { mutagen }\end{array}$ & $\begin{array}{l}\text { Mutagenicity } \\
\text { Positive }\end{array}$ & Mutagen & Mutagen & Mutagen \\
\hline 8 & 4-Aminobiphenyl & $\begin{array}{l}\text { Structural alert for } S . \text { typhimurium } \\
\text { mutagenicity; Potensial } S \text {. } \\
\text { typhimurium TA } 100 \text { mutagen }\end{array}$ & $\begin{array}{l}\text { Mutagenicity } \\
\text { Positive }\end{array}$ & Mutagen & Mutagen & Mutagen \\
\hline 9 & $\begin{array}{l}\text { 4-(N- } \\
\text { Nitrosomethyl- } \\
\text { amino)-1-(3- } \\
\text { pyridyl)-1-butanone }\end{array}$ & $\begin{array}{l}\text { Structural alert for S. typhimurium } \\
\text { mutagenicity }\end{array}$ & $\begin{array}{l}\text { Mutagenicity } \\
\text { Positive }\end{array}$ & Mutagen & Mutagen & Mutagen \\
\hline
\end{tabular}


Table 2. Mutagenicity Prediction for Test Chemical

\begin{tabular}{|c|c|c|c|c|c|c|}
\hline No. & Compound & Toxtree & T.E.S.T. & $\begin{array}{l}\text { VEGA } \\
\text { Benigni/Bossa }\end{array}$ & CAESAR & SARPy \\
\hline 1 & p-Tolylacetaldehyde & $\begin{array}{l}\text { Structural alert for S. typhimurium } \\
\text { mutagenicity }\end{array}$ & $\begin{array}{l}\text { Mutagenicity } \\
\text { Positive }\end{array}$ & Mutagen & Non Mutagen & $\begin{array}{l}\text { Non } \\
\text { Mutagen }\end{array}$ \\
\hline 2 & $\begin{array}{l}\text { p-Isopropylphenyl- } \\
\text { acetaldehyde }\end{array}$ & $\begin{array}{l}\text { Structural alert for S. typhimurium } \\
\text { mutagenicity }\end{array}$ & $\begin{array}{l}\text { Mutagenicity } \\
\text { Negative }\end{array}$ & Mutagen & Non Mutagen & $\begin{array}{l}\text { Non } \\
\text { Mutagen }\end{array}$ \\
\hline 3 & Cinnamaldehyde & $\begin{array}{l}\text { No alert for S.typhimurium } \\
\text { mutagenicity; Unlikely to be a } S \text {. } \\
\text { typhimurium TA } 100 \text { mutagen }\end{array}$ & $\begin{array}{l}\text { Mutagenicity } \\
\text { Positive }\end{array}$ & Non Mutagen & Non Mutagen & $\begin{array}{l}\text { Non } \\
\text { Mutagen }\end{array}$ \\
\hline 4 & $\begin{array}{l}\text { 3-(p-isopropylphenyl) } \\
\text { propionaldehyde }\end{array}$ & $\begin{array}{l}\text { No alert for } S \text {. typhimurium } \\
\text { mutagenicity; Unlikely to be a } S \text {. } \\
\text { typhimurium TA100 mutagen }\end{array}$ & $\begin{array}{l}\text { Mutagenicity } \\
\text { Negative }\end{array}$ & Mutagen & Non Mutagen & $\begin{array}{l}\text { Non } \\
\text { Mutagen }\end{array}$ \\
\hline 5 & $\begin{array}{l}\text { alpha-Amylcinnamal- } \\
\text { dehyde dimethyl acetal }\end{array}$ & $\begin{array}{l}\text { No alert for } S . \text { typhimurium } \\
\text { mutagenicity }\end{array}$ & $\begin{array}{l}\text { Mutagenicity } \\
\text { Negative }\end{array}$ & Non Mutagen & Non Mutagen & $\begin{array}{l}\text { Non } \\
\text { Mutagen }\end{array}$ \\
\hline 6 & $\begin{array}{l}\text { p-Methylcinnamal- } \\
\text { dehyde }\end{array}$ & $\begin{array}{l}\text { No alert for } S \text {. typhimurium } \\
\text { mutagenicity; Unlikely to be a } S \text {. } \\
\text { typhimurium TA100 mutagen }\end{array}$ & $\begin{array}{l}\text { Mutagenicity } \\
\text { Positive }\end{array}$ & Mutagen & Non Mutagen & $\begin{array}{l}\text { Non } \\
\text { Mutagen }\end{array}$ \\
\hline 7 & $\begin{array}{l}\text { alpha- } \\
\text { Methylcinnamaldehyde }\end{array}$ & $\begin{array}{l}\text { No alert for } S \text {. typhimurium } \\
\text { mutagenicity; Unlikely to be a } S \text {. } \\
\text { typhimurium TA } 100 \text { mutagen }\end{array}$ & $\begin{array}{l}\text { Mutagenicity } \\
\text { Positive }\end{array}$ & Mutagen & Non Mutagen & $\begin{array}{l}\text { Non } \\
\text { Mutagen }\end{array}$ \\
\hline 8 & $\begin{array}{l}\text { alpha-Buthylcinnamal- } \\
\text { dehyde }\end{array}$ & $\begin{array}{l}\text { No alert for } S \text {. typhimurium } \\
\text { mutagenicity; Unlikely to be a } S \text {. } \\
\text { typhimurium TA } 100 \text { mutagen }\end{array}$ & $\begin{array}{l}\text { Mutagenicity } \\
\text { Negative }\end{array}$ & Non Mutagen & Non Mutagen & $\begin{array}{l}\text { Non } \\
\text { Mutagen }\end{array}$ \\
\hline 9 & $\begin{array}{l}\text { alpha-Amylcinnamal- } \\
\text { dehyde }\end{array}$ & $\begin{array}{l}\text { No alert for } S \text {. typhimurium } \\
\text { mutagenicity; Unlikely to be a } S \text {. } \\
\text { typhimurium TA } 100 \text { mutagen }\end{array}$ & $\begin{array}{l}\text { Mutagenicity } \\
\text { Negative }\end{array}$ & Non Mutagen & Non Mutagen & $\begin{array}{l}\text { Non } \\
\text { Mutagen }\end{array}$ \\
\hline 10 & $\begin{array}{l}\text { alpha-Hexylcinnamal- } \\
\text { dehyde }\end{array}$ & $\begin{array}{l}\text { No alert for } S \text {. typhimurium } \\
\text { mutagenicity; Unlikely to be a } S \text {. } \\
\text { typhimurium TA100 mutagen }\end{array}$ & $\begin{array}{l}\text { Mutagenicity } \\
\text { Negative }\end{array}$ & Non Mutagen & Non Mutagen & $\begin{array}{l}\text { Non } \\
\text { Mutagen }\end{array}$ \\
\hline 11 & $\begin{array}{l}\text { p-Methoxycinnamal- } \\
\text { dehyde }\end{array}$ & $\begin{array}{l}\text { No alert for } S \text {. typhimurium } \\
\text { mutagenicity; Unlikely to be a } S \text {. } \\
\text { typhimurium TA100 mutagen }\end{array}$ & $\begin{array}{l}\text { Mutagenicity } \\
\text { Positive }\end{array}$ & Mutagen & Non Mutagen & $\begin{array}{l}\text { Non } \\
\text { Mutagen }\end{array}$ \\
\hline 12 & $\begin{array}{l}\text { o-Methoxycinnamal- } \\
\text { dehyde }\end{array}$ & $\begin{array}{l}\text { No alert for } S \text {. typhimurium } \\
\text { mutagenicity; Unlikely to be a } S \text {. } \\
\text { typhimurium TA } 100 \text { mutagen }\end{array}$ & $\begin{array}{l}\text { Mutagenicity } \\
\text { Positive }\end{array}$ & Mutagen & Non Mutagen & $\begin{array}{l}\text { Non } \\
\text { Mutagen }\end{array}$ \\
\hline 13 & $\begin{array}{l}\text { p-Methoxy-alpha- } \\
\text { methylcinnamaldehyde }\end{array}$ & $\begin{array}{l}\text { No alert for } S \text {. typhimurium } \\
\text { mutagenicity; Unlikely to be a } S \text {. } \\
\text { typhimurium TA100 mutagen }\end{array}$ & $\begin{array}{l}\text { Mutagenicity } \\
\text { Positive }\end{array}$ & Mutagen & Non Mutagen & $\begin{array}{l}\text { Non } \\
\text { Mutagen }\end{array}$ \\
\hline 14 & $\begin{array}{l}\text { 2-Methyl-4-phenylbu- } \\
\text { tyraldehyde }\end{array}$ & $\begin{array}{l}\text { Structural alert for S. typhimurium } \\
\text { mutagenicity }\end{array}$ & $\begin{array}{l}\text { Mutagenicity } \\
\text { Negative }\end{array}$ & Mutagen & Non Mutagen & $\begin{array}{l}\text { Non } \\
\text { Mutagen }\end{array}$ \\
\hline 15 & $\begin{array}{l}\text { 3-Methyl-2-phenylbu- } \\
\text { tyraldehyde }\end{array}$ & $\begin{array}{l}\text { Structural alert for } S \text {. typhimurium } \\
\text { mutagenicity }\end{array}$ & $\begin{array}{l}\text { Mutagenicity } \\
\text { Negative }\end{array}$ & Mutagen & Non Mutagen & $\begin{array}{l}\text { Non } \\
\text { Mutagen }\end{array}$ \\
\hline 16 & $\begin{array}{l}\text { 2-Methyl-3-(p- } \\
\text { isopropylphenyl) } \\
\text { propionaldehyde }\end{array}$ & $\begin{array}{l}\text { Structural alert for S. typhimurium } \\
\text { mutagenicity }\end{array}$ & $\begin{array}{l}\text { Mutagenicity } \\
\text { Negative }\end{array}$ & Mutagen & Non Mutagen & $\begin{array}{l}\text { Non } \\
\text { Mutagen }\end{array}$ \\
\hline
\end{tabular}


Table 2. (continued)

\begin{tabular}{|c|c|c|c|c|c|c|}
\hline 17 & $\begin{array}{l}\text { 2-Methyl-3-tolyl- } \\
\text { propionaldehyde }\end{array}$ & $\begin{array}{l}\text { Structural alert for } S \text {. } \\
\text { typhimurium mutagenicity }\end{array}$ & $\begin{array}{l}\text { Mutagenicity } \\
\text { Negative }\end{array}$ & Mutagen & Non Mutagen & $\begin{array}{l}\text { Non } \\
\text { Mutagen }\end{array}$ \\
\hline 18 & $\begin{array}{l}\text { 2-Phenylpropional- } \\
\text { dehyde }\end{array}$ & $\begin{array}{l}\text { Structural alert for } S \text {. } \\
\text { typhimurium mutagenicity }\end{array}$ & $\begin{array}{l}\text { Mutagenicity } \\
\text { Negative }\end{array}$ & Mutagen & Non Mutagen & $\begin{array}{l}\text { Non } \\
\text { Mutagen }\end{array}$ \\
\hline 19 & $\begin{array}{l}\text { 2-Phenylpropional- } \\
\text { dehyde dimethyl acetal }\end{array}$ & $\begin{array}{l}\text { No alert for } S \text {. typhimurium } \\
\text { mutagenicity }\end{array}$ & $\begin{array}{l}\text { Mutagenicity } \\
\text { Negative }\end{array}$ & Non Mutagen & Non Mutagen & $\begin{array}{l}\text { Non } \\
\text { Mutagen }\end{array}$ \\
\hline 20 & $\begin{array}{l}\text { 2-(p-Tolyl)propional- } \\
\text { dehyde }\end{array}$ & $\begin{array}{l}\text { Structural alert for } S \text {. } \\
\text { typhimurium mutagenicity }\end{array}$ & $\begin{array}{l}\text { Mutagenicity } \\
\text { Negative }\end{array}$ & Mutagen & Non Mutagen & $\begin{array}{l}\text { Non } \\
\text { Mutagen }\end{array}$ \\
\hline 21 & Benzaldehyde & $\begin{array}{l}\text { Structural alert for } S \text {. } \\
\text { typhimurium mutagenicity }\end{array}$ & $\begin{array}{l}\text { Mutagenicity } \\
\text { Negative }\end{array}$ & Mutagen & Non Mutagen & $\begin{array}{l}\text { Non } \\
\text { Mutagen }\end{array}$ \\
\hline 22 & $\begin{array}{l}\text { Benzaldehyde dimethyl } \\
\text { acetal }\end{array}$ & $\begin{array}{l}\text { No alert for } S \text {. typhimurium } \\
\text { mutagenicity }\end{array}$ & $\begin{array}{l}\text { Mutagenicity } \\
\text { Negative }\end{array}$ & Non Mutagen & Non Mutagen & $\begin{array}{l}\text { Non } \\
\text { Mutagen }\end{array}$ \\
\hline 23 & $\begin{array}{l}\text { Benzaldehydeglyceryl- } \\
\text { acetal }\end{array}$ & $\begin{array}{l}\text { No alert for } S \text {. typhimurium } \\
\text { mutagenicity }\end{array}$ & $\begin{array}{l}\text { Mutagenicity } \\
\text { Negative }\end{array}$ & Non Mutagen & Mutagen & $\begin{array}{l}\text { Non } \\
\text { Mutagen }\end{array}$ \\
\hline 24 & $\begin{array}{l}\text { Benzaldehyde } \\
\text { propylene glycol acetal }\end{array}$ & $\begin{array}{l}\text { No alert for } S \text {. typhimurium } \\
\text { mutagenicity }\end{array}$ & $\begin{array}{l}\text { Mutagenicity } \\
\text { Negative }\end{array}$ & Non Mutagen & Mutagen & $\begin{array}{l}\text { Non } \\
\text { Mutagen }\end{array}$ \\
\hline 25 & 4-Ethylbenzaldehyde & $\begin{array}{l}\text { Structural alert for } S \text {. } \\
\text { typhimurium mutagenicity }\end{array}$ & $\begin{array}{l}\text { Mutagenicity } \\
\text { Negative }\end{array}$ & Mutagen & Non Mutagen & $\begin{array}{l}\text { Non } \\
\text { Mutagen }\end{array}$ \\
\hline 26 & Tolualdehyde & $\begin{array}{l}\text { Structural alert for } S \text {. } \\
\text { typhimurium mutagenicity }\end{array}$ & $\begin{array}{l}\text { Mutagenicity } \\
\text { Negative }\end{array}$ & Mutagen & Non Mutagen & $\begin{array}{l}\text { Non } \\
\text { Mutagen }\end{array}$ \\
\hline 27 & $\begin{array}{l}\text { Tolualdehydeglyceryl- } \\
\text { acetal }\end{array}$ & $\begin{array}{l}\text { No alert for } S \text {. typhimurium } \\
\text { mutagenicity }\end{array}$ & $\begin{array}{l}\text { Mutagenicity } \\
\text { Negative }\end{array}$ & Non Mutagen & Non Mutagen & $\begin{array}{l}\text { Non } \\
\text { Mutagen }\end{array}$ \\
\hline 28 & Cuminaldehyde & $\begin{array}{l}\text { Structural alert for } S \text {. } \\
\text { typhimurium mutagenicity }\end{array}$ & $\begin{array}{l}\text { Mutagenicity } \\
\text { Negative }\end{array}$ & Mutagen & Non Mutagen & $\begin{array}{l}\text { Non } \\
\text { Mutagen }\end{array}$ \\
\hline 29 & $\begin{array}{l}\text { 2,4-Dimethylbenzal- } \\
\text { dehyde }\end{array}$ & $\begin{array}{l}\text { Structural alert for } S \text {. } \\
\text { typhimurium mutagenicity }\end{array}$ & $\begin{array}{l}\text { Mutagenicity } \\
\text { Negative }\end{array}$ & Mutagen & Non Mutagen & $\begin{array}{l}\text { Non } \\
\text { Mutagen }\end{array}$ \\
\hline 30 & Veratraldehyde & $\begin{array}{l}\text { Structural alert for } S \text {. } \\
\text { typhimurium mutagenicity }\end{array}$ & $\begin{array}{l}\text { Mutagenicity } \\
\text { Negative }\end{array}$ & Mutagen & Non Mutagen & $\begin{array}{l}\text { Non } \\
\text { Mutagen }\end{array}$ \\
\hline 31 & $\begin{array}{l}\text { p-Methoxybenzal- } \\
\text { dehyde }\end{array}$ & $\begin{array}{l}\text { Structural alert for } S \text {. } \\
\text { typhimurium mutagenicity }\end{array}$ & $\begin{array}{l}\text { Mutagenicity } \\
\text { Negative }\end{array}$ & Mutagen & Non Mutagen & $\begin{array}{l}\text { Non } \\
\text { Mutagen }\end{array}$ \\
\hline 32 & p-Ethoxybenzaldehyde & $\begin{array}{l}\text { Structural alert for } S \text {. } \\
\text { typhimurium mutagenicity }\end{array}$ & $\begin{array}{l}\text { Mutagenicity } \\
\text { Negative }\end{array}$ & Mutagen & Non Mutagen & $\begin{array}{l}\text { Non } \\
\text { Mutagen }\end{array}$ \\
\hline 33 & Salicylaldehyde & $\begin{array}{l}\text { Structural alert for } S \text {. } \\
\text { typhimurium mutagenicity }\end{array}$ & $\begin{array}{l}\text { Mutagenicity } \\
\text { Negative }\end{array}$ & Mutagen & Non Mutagen & $\begin{array}{l}\text { Non } \\
\text { Mutagen }\end{array}$ \\
\hline 34 & $\begin{array}{l}\text { 2-Hydroxy-4-methyl- } \\
\text { benzaldehyde }\end{array}$ & $\begin{array}{l}\text { Structural alert for } S \text {. } \\
\text { typhimurium mutagenicity }\end{array}$ & $\begin{array}{l}\text { Mutagenicity } \\
\text { Negative }\end{array}$ & Mutagen & Non Mutagen & $\begin{array}{l}\text { Non } \\
\text { Mutagen }\end{array}$ \\
\hline 35 & $\begin{array}{l}\text { 4-Hydroxybenzal- } \\
\text { dehyde }\end{array}$ & $\begin{array}{l}\text { Structural alert for } S \text {. } \\
\text { typhimurium mutagenicity }\end{array}$ & $\begin{array}{l}\text { Mutagenicity } \\
\text { Negative }\end{array}$ & Mutagen & Non Mutagen & $\begin{array}{l}\text { Non } \\
\text { Mutagen }\end{array}$ \\
\hline 36 & $\begin{array}{l}\text { o-Methoxybenzal- } \\
\text { dehyde }\end{array}$ & $\begin{array}{l}\text { Structural alert for } S \text {. } \\
\text { typhimurium mutagenicity }\end{array}$ & $\begin{array}{l}\text { Mutagenicity } \\
\text { Negative }\end{array}$ & Mutagen & Non Mutagen & $\begin{array}{l}\text { Non } \\
\text { Mutagen }\end{array}$ \\
\hline 37 & $\begin{array}{l}\text { 4-Hydroxy-3,5-dime- } \\
\text { thoxybenzaldehyde }\end{array}$ & $\begin{array}{l}\text { Structural alert for } S \text {. } \\
\text { typhimurium mutagenicity }\end{array}$ & $\begin{array}{l}\text { Mutagenicity } \\
\text { Negative }\end{array}$ & Mutagen & Non Mutagen & $\begin{array}{l}\text { Non } \\
\text { Mutagen }\end{array}$ \\
\hline 38 & $\begin{array}{l}\text { 2-Benzofurancarboxal- } \\
\text { dehyde }\end{array}$ & $\begin{array}{l}\text { No alert for } S . \text { typhimurium } \\
\text { mutagenicity }\end{array}$ & $\begin{array}{l}\text { Mutagenicity } \\
\text { Positive }\end{array}$ & Non Mutagen & Mutagen & $\begin{array}{l}\text { Non } \\
\text { Mutagen }\end{array}$ \\
\hline 39 & $\begin{array}{l}\text { 2-Furfurylidenebu- } \\
\text { tyraldehyde }\end{array}$ & $\begin{array}{l}\text { No alert for } S \text {. typhimurium } \\
\text { mutagenicity; Unlikely to be a } \\
\text { S. typhimurium TA } 100 \text { mutagen }\end{array}$ & $\begin{array}{l}\text { Mutagenicity } \\
\text { Negative }\end{array}$ & Mutagen & Non Mutagen & $\begin{array}{l}\text { Non } \\
\text { Mutagen }\end{array}$ \\
\hline
\end{tabular}


Table 2. (continued)

\begin{tabular}{|c|c|c|c|c|c|c|}
\hline 40 & 2-Methylfuran & $\begin{array}{l}\text { No alert for } S \text {. typhimurium } \\
\text { mutagenicity }\end{array}$ & $\begin{array}{l}\text { Mutagenicity } \\
\text { Negative }\end{array}$ & Non Mutagen & Non Mutagen & $\begin{array}{l}\text { Non } \\
\text { Mutagen }\end{array}$ \\
\hline 41 & 2,5-Dimethylfuran & $\begin{array}{l}\text { No alert for } S \text {. typhimurium } \\
\text { mutagenicity }\end{array}$ & $\begin{array}{l}\text { Mutagenicity } \\
\text { Negative }\end{array}$ & Non Mutagen & Non Mutagen & $\begin{array}{l}\text { Non } \\
\text { Mutagen }\end{array}$ \\
\hline 42 & 2-Ethylfuran & $\begin{array}{l}\text { No alert for } S \text {. typhimurium } \\
\text { mutagenicity }\end{array}$ & $\begin{array}{l}\text { Mutagenicity } \\
\text { Negative }\end{array}$ & Non Mutagen & Non Mutagen & $\begin{array}{l}\text { Non } \\
\text { Mutagen }\end{array}$ \\
\hline 43 & 2-Butylfuran & $\begin{array}{l}\text { No alert for } S \text {. typhimurium } \\
\text { mutagenicity }\end{array}$ & $\begin{array}{l}\text { Mutagenicity } \\
\text { Negative }\end{array}$ & Non Mutagen & Non Mutagen & $\begin{array}{l}\text { Non } \\
\text { Mutagen }\end{array}$ \\
\hline 44 & 2-Pentylfuran & $\begin{array}{l}\text { No alert for } S \text {. typhimurium } \\
\text { mutagenicity }\end{array}$ & $\begin{array}{l}\text { Mutagenicity } \\
\text { Negative }\end{array}$ & Non Mutagen & Non Mutagen & $\begin{array}{l}\text { Non } \\
\text { Mutagen }\end{array}$ \\
\hline 45 & 2-Heptylfuran & $\begin{array}{l}\text { No alert for } S \text {. typhimurium } \\
\text { mutagenicity }\end{array}$ & $\begin{array}{l}\text { Mutagenicity } \\
\text { Negative }\end{array}$ & Non Mutagen & Non Mutagen & $\begin{array}{l}\text { Non } \\
\text { Mutagen }\end{array}$ \\
\hline 46 & 2-Decylfuran & $\begin{array}{l}\text { No alert for } S \text {. typhimurium } \\
\text { mutagenicity }\end{array}$ & $\begin{array}{l}\text { Mutagenicity } \\
\text { Negative }\end{array}$ & Non Mutagen & Non Mutagen & $\begin{array}{l}\text { Non } \\
\text { Mutagen }\end{array}$ \\
\hline 47 & 2-Butyrylfuran & $\begin{array}{l}\text { No alert for } S . \text { typhimurium } \\
\text { mutagenicity }\end{array}$ & $\begin{array}{l}\text { Mutagenicity } \\
\text { Negative }\end{array}$ & Non Mutagen & Non Mutagen & $\begin{array}{l}\text { Non } \\
\text { Mutagen }\end{array}$ \\
\hline 48 & 2-Pentanoylfuran & $\begin{array}{l}\text { No alert for } S . \text { typhimurium } \\
\text { mutagenicity }\end{array}$ & $\begin{array}{l}\text { Mutagenicity } \\
\text { Negative }\end{array}$ & Non Mutagen & Non Mutagen & $\begin{array}{l}\text { Non } \\
\text { Mutagen }\end{array}$ \\
\hline 49 & $\begin{array}{l}\text { 3-Methyl-2-(3- } \\
\text { methylbut-2-enyl)- } \\
\text { furan }\end{array}$ & $\begin{array}{l}\text { No alert for } S . \text { typhimurium } \\
\text { mutagenicity }\end{array}$ & $\begin{array}{l}\text { Mutagenicity } \\
\text { Negative }\end{array}$ & Non Mutagen & Non Mutagen & $\begin{array}{l}\text { Non } \\
\text { Mutagen }\end{array}$ \\
\hline 50 & $\begin{array}{l}\text { 2,3- } \\
\text { Dimethylbenzofuran }\end{array}$ & $\begin{array}{l}\text { No alert for } S . \text { typhimurium } \\
\text { mutagenicity }\end{array}$ & $\begin{array}{l}\text { Mutagenicity } \\
\text { Negative }\end{array}$ & Non Mutagen & Non Mutagen & $\begin{array}{l}\text { Non } \\
\text { Mutagen }\end{array}$ \\
\hline 51 & 2,4-Difurfurylfuran & $\begin{array}{l}\text { No alert for } S . \text { typhimurium } \\
\text { mutagenicity }\end{array}$ & $\begin{array}{l}\text { Mutagenicity } \\
\text { Positive }\end{array}$ & Non Mutagen & Non Mutagen & $\begin{array}{l}\text { Non } \\
\text { Mutagen }\end{array}$ \\
\hline 52 & $\begin{array}{l}\text { 2-Acetyl-5- } \\
\text { methylfuran }\end{array}$ & $\begin{array}{l}\text { No alert for } S \text {. typhimurium } \\
\text { mutagenicity }\end{array}$ & $\begin{array}{l}\text { Mutagenicity } \\
\text { Positive }\end{array}$ & Non Mutagen & Mutagen & $\begin{array}{l}\text { Non } \\
\text { Mutagen }\end{array}$ \\
\hline 53 & $\begin{array}{l}\text { 2-Acetyl-3,5- } \\
\text { dimethylfuran }\end{array}$ & $\begin{array}{l}\text { No alert for } S . \text { typhimurium } \\
\text { mutagenicity }\end{array}$ & $\begin{array}{l}\text { Mutagenicity } \\
\text { Negative }\end{array}$ & Non Mutagen & Non Mutagen & $\begin{array}{l}\text { Non } \\
\text { Mutagen }\end{array}$ \\
\hline 54 & $\begin{array}{l}\text { 3-Acetyl-2,5- } \\
\text { dimethylfuran }\end{array}$ & $\begin{array}{l}\text { No alert for } S \text {. typhimurium } \\
\text { mutagenicity }\end{array}$ & $\begin{array}{l}\text { Mutagenicity } \\
\text { Negative }\end{array}$ & Non Mutagen & Non Mutagen & $\begin{array}{l}\text { Non } \\
\text { Mutagen }\end{array}$ \\
\hline
\end{tabular}

Table 3. Carcinogenicity Prediction for Control Chemical

\begin{tabular}{llllc}
\hline No. & Compound & Toxtree & VEGA & Benigni/Bossa \\
\hline 1 & Benzo[a]pyrene & Structural alert for genotoxic carcinogenicity & Carcinogen & Carcinogen \\
2 & Benzidine & Structural alert for genotoxic carcinogenicity & Carcinogen & Carcinogen \\
3 & Bis (chloromethyl) ether & Structural alert for genotoxic carcinogenicity & Carcinogen & Carcinogen \\
4 & Chloromethyl methyl ether & Structural alert for genotoxic carcinogenicity & Carcinogen & Carcinogen \\
5 & Ethylene oxide & Structural alert for genotoxic carcinogenicity & Carcinogen & Carcinogen \\
6 & N'-Nitrosonornicotine & Structural alert for genotoxic carcinogenicity & Non Carcinogen* & Carcinogen \\
7 & 2-Naphthylamine & $\begin{array}{l}\text { Structural alert for genotoxic carcinogenicity; Potential } \\
\text { carcinogen }\end{array}$ & Carcinogen & Carcinogen \\
8 & 4-Aminobiphenyl & $\begin{array}{l}\text { Structural alert for genotoxic carcinogenicity; Potential } \\
\text { carcinogen }\end{array}$ & Carcinogen & Carcinogen \\
9 & $\begin{array}{l}\text { 4-(N-Nitrosomethylamino)-1- } \\
\text { (3-pyridyl)-1-butanone }\end{array}$ & $\begin{array}{l}\text { Structural alert for genotoxic carcinogenicity } \\
\text { Carcinogen }\end{array}$ & Carcinogen \\
\hline
\end{tabular}


Table 4. Carcinogenicity Prediction for Test Chemical

\begin{tabular}{|c|c|c|c|c|}
\hline No. & Compound & TOXTREE & $\begin{array}{l}\text { VEGA } \\
\text { Benigni/Bossa }\end{array}$ & CAESAR \\
\hline 1 & p-Tolylacetaldehyde & Structural alert for genotoxic carcinogenicity & Carcinogenic & Non Carcinogenic \\
\hline 2 & p-Isopropylphenylacetaldehyde & Structural alert for genotoxic carcinogenicity & Carcinogenic & Carcinogenic \\
\hline 3 & Cinnamaldehyde & $\begin{array}{l}\text { Negative for genotoxic carcinogenicity and non } \\
\text { genotoxic carcinogenicity }\end{array}$ & Non Carcinogenic & Non Carcinogenic \\
\hline 4 & $\begin{array}{l}\text { 3-(p-isopropylphenyl) } \\
\text { propionaldehyde }\end{array}$ & Structural alert for genotoxic carcinogenicity & Carcinogenic & Carcinogenic \\
\hline 5 & $\begin{array}{l}\text { alpha-Amylcinnamaldehyde } \\
\text { dimethyl acetal }\end{array}$ & $\begin{array}{l}\text { Negative for genotoxic carcinogenicity and non } \\
\text { genotoxic carcinogenicity }\end{array}$ & Non Carcinogenic & Non Carcinogenic \\
\hline 6 & p-Methylcinnamaldehyde & $\begin{array}{l}\text { Negative for genotoxic carcinogenicity and non } \\
\text { genotoxic carcinogenicity }\end{array}$ & Carcinogenic & Non Carcinogenic \\
\hline 7 & alpha-Methylcinnamaldehyde & $\begin{array}{l}\text { Negative for genotoxic carcinogenicity and non } \\
\text { genotoxic carcinogenicity }\end{array}$ & Carcinogenic & Non Carcinogenic \\
\hline 8 & alpha-Buthylcinnamaldehyde & $\begin{array}{l}\text { Negative for genotoxic carcinogenicity and non } \\
\text { genotoxic carcinogenicity }\end{array}$ & Non Carcinogenic & Non Carcinogenic \\
\hline 9 & alpha-Amylcinnamaldehyde & $\begin{array}{l}\text { Negative for genotoxic carcinogenicity and non } \\
\text { genotoxic carcinogenicity }\end{array}$ & Carcinogenic & Non Carcinogenic \\
\hline 10 & alpha-Hexylcinnamaldehyde & $\begin{array}{l}\text { Negative for genotoxic carcinogenicity and non } \\
\text { genotoxic carcinogenicity }\end{array}$ & Non Carcinogenic & Non Carcinogenic \\
\hline 11 & p-Methoxycinnamaldehyde & $\begin{array}{l}\text { Negative for genotoxic carcinogenicity and non } \\
\text { genotoxic carcinogenicity }\end{array}$ & Carcinogenic & Non Carcinogenic \\
\hline 12 & o-Methoxycinnamaldehyde & $\begin{array}{l}\text { Negative for genotoxic carcinogenicity and non } \\
\text { genotoxic carcinogenicity }\end{array}$ & Carcinogenic & Carcinogenic \\
\hline 13 & $\begin{array}{l}\text { p-Methoxy-alpha- } \\
\text { methylcinnamaldehyde }\end{array}$ & $\begin{array}{l}\text { Negative for genotoxic carcinogenicity and non } \\
\text { genotoxic carcinogenicity }\end{array}$ & Carcinogenic & Non Carcinogenic \\
\hline 14 & 2-Methyl-4-phenylbutyraldehyde & Structural alert for genotoxic carcinogenicity & Carcinogenic & Non Carcinogenic \\
\hline 15 & 3-Methyl-2-phenylbutyraldehyde & Structural alert for genotoxic carcinogenicity & Carcinogenic & Carcinogenic \\
\hline 16 & $\begin{array}{l}\text { 2-Methyl-3-(p-isopropylphenyl) } \\
\text { propionaldehyde }\end{array}$ & Structural alert for genotoxic carcinogenicity & Carcinogenic & Carcinogenic \\
\hline 17 & 2-Methyl-3-tolylpropionaldehyde & Structural alert for genotoxic carcinogenicity & Carcinogenic & Non Carcinogenic \\
\hline 18 & 2-Phenylpropionaldehyde & Structural alert for genotoxic carcinogenicity & Carcinogenic & Non Carcinogenic \\
\hline 19 & $\begin{array}{l}\text { 2-Phenylpropionaldehyde dimethyl } \\
\text { acetal }\end{array}$ & $\begin{array}{l}\text { Negative for genotoxic carcinogenicity and non } \\
\text { genotoxic carcinogenicity }\end{array}$ & Non Carcinogenic & Carcinogenic \\
\hline 20 & 2-(p-Tolyl)propionaldehyde & Structural alert for genotoxic carcinogenicity & Carcinogenic & Carcinogenic \\
\hline 21 & Benzaldehyde & Structural alert for genotoxic carcinogenicity & Carcinogenic & Non Carcinogenic \\
\hline 22 & Benzaldehyde dimethyl acetal & $\begin{array}{l}\text { Negative for genotoxic carcinogenicity and non } \\
\text { genotoxic carcinogenicity }\end{array}$ & Non Carcinogenic & Carcinogenic \\
\hline 23 & Benzaldehydeglycerylacetal & $\begin{array}{l}\text { Negative for genotoxic carcinogenicity and non } \\
\text { genotoxic carcinogenicity }\end{array}$ & Non Carcinogenic & Non Carcinogenic \\
\hline
\end{tabular}


Table 4. (continued) acetal

4-Ethylbenzaldehyde

Tolualdehyde

27

Tolualdehydeglycerylacetal

Cuminaldehyde

\section{2,4-Dimethylbenzaldehyde}

Veratraldehyde

31

p-Methoxybenzaldehyde

p-Ethoxybenzaldehyde
Salicylaldehyde

2-Hydroxy-4-methylbenzaldehyde

4-Hydroxybenzaldehyde

o-Methoxybenzaldehyde

4-Hydroxy-3,5-

dimethoxybenzaldehyde

2-Benzofurancarboxaldehyde

2-Furfurylidenebutyraldehyde

2-Methylfuran

\section{2,5-Dimethylfuran}

\section{2-Ethylfuran}

\section{2-Butylfuran}

\section{2-Heptylfuran}

2-Decylfuran

2-Pentanoylfuran
Negative for genotoxic carcinogenicity and non genotoxic carcinogenicity

Structural alert for genotoxic carcinogenicity

Structural alert for genotoxic carcinogenicity

Negative for genotoxic carcinogenicity and non genotoxic carcinogenicity

Structural alert for genotoxic carcinogenicity

Structural alert for genotoxic carcinogenicity

Structural alert for genotoxic carcinogenicity

Structural alert for genotoxic carcinogenicity

Structural alert for genotoxic carcinogenicity

Structural alert for genotoxic carcinogenicity

Structural alert for genotoxic carcinogenicity

Structural alert for genotoxic carcinogenicity

Structural alert for genotoxic carcinogenicity

Structural alert for genotoxic carcinogenicity

Negative for genotoxic carcinogenicity and non genotoxic carcinogenicity

Negative for genotoxic carcinogenicity and non genotoxic carcinogenicity

Negative for genotoxic carcinogenicity and non genotoxic carcinogenicity

Negative for genotoxic carcinogenicity and non genotoxic carcinogenicity

Negative for genotoxic carcinogenicity and non genotoxic carcinogenicity

Negative for genotoxic carcinogenicity and non genotoxic carcinogenicity

Negative for genotoxic carcinogenicity and non genotoxic carcinogenicity

Negative for genotoxic carcinogenicity and non genotoxic carcinogenicity

Negative for genotoxic carcinogenicity and non genotoxic carcinogenicity

Negative for genotoxic carcinogenicity and non genotoxic carcinogenicity

Negative for genotoxic carcinogenicity and non genotoxic carcinogenicity
Non Carcinogenic

Carcinogenic

Carcinogenic

Non Carcinogenic

Carcinogenic

Non Carcinogenic

Non Carcinogenic

Carcinogenic

Carcinogenic

Carcinogenic

Carcinogenic

Carcinogenic

Carcinogenic

Carcinogenic

Non Carcinogenic

Non Carcinogenic

Carcinogenic

Non Carcinogenic

Carcinogenic

Carcinogenic

Carcinogenic

Carcinogenic

Carcinogenic

Non Carcinogenic

Carcinogenic

Carcinogenic

Carcinogenic

Non Carcinogenic

Non Carcinogenic

Non Carcinogenic

Carcinogenic

Non Carcinogenic

Non Carcinogenic

Carcinogenic

Non Carcinogenic

Non Carcinogenic

Non Carcinogenic

Non Carcinogenic

Non Carcinogenic

Non Carcinogenic

Non Carcinogenic

Non Carcinogenic

Non Carcinogenic

Non Carcinogenic

Non Carcinogenic

Non Carcinogenic

Non Carcinogenic

Non Carcinogenic

Non Carcinogenic Carcinogenic 
Table 4. (continued)

\begin{tabular}{lllll}
\hline 49 & $\begin{array}{l}\text { 3-Methyl-2-(3-methylbut-2-enyl)- } \\
\text { furan }\end{array}$ & $\begin{array}{l}\text { Negative for genotoxic carcinogenicity and non } \\
\text { genotoxic carcinogenicity }\end{array}$ & Non Carcinogenic & Non Carcinogenic \\
50 & 2,3-Dimethylbenzofuran & $\begin{array}{l}\text { Negative for genotoxic carcinogenicity and non } \\
\text { genotoxic carcinogenicity }\end{array}$ & Non Carcinogenic & Carcinogenic \\
51 & 2,4-Difurfurylfuran & $\begin{array}{l}\text { Negative for genotoxic carcinogenicity and non } \\
\text { genotoxic carcinogenicity }\end{array}$ & Non Carcinogenic & Carcinogenic \\
$52 \quad$ 2-Acetyl-5-methylfuran & $\begin{array}{l}\text { Negative for genotoxic carcinogenicity and non } \\
\text { genotoxic carcinogenicity }\end{array}$ & Non Carcinogenic & Carcinogenic \\
& & $\begin{array}{l}\text { Negative for genotoxic carcinogenicity and non } \\
\text { genotoxic carcinogenicity }\end{array}$ & Non Carcinogenic & Carcinogenic \\
53 & 2-Acetyl-3,5-dimethylfuran & $\begin{array}{l}\text { Negative for genotoxic carcinogenicity and non } \\
\text { genotoxic carcinogenicity }\end{array}$ & Non Carcinogenic & Carcinogenic \\
\hline
\end{tabular}

